\title{
mIOROSCOPY TODAY
}

maY | 998

ISSUE \#98-4

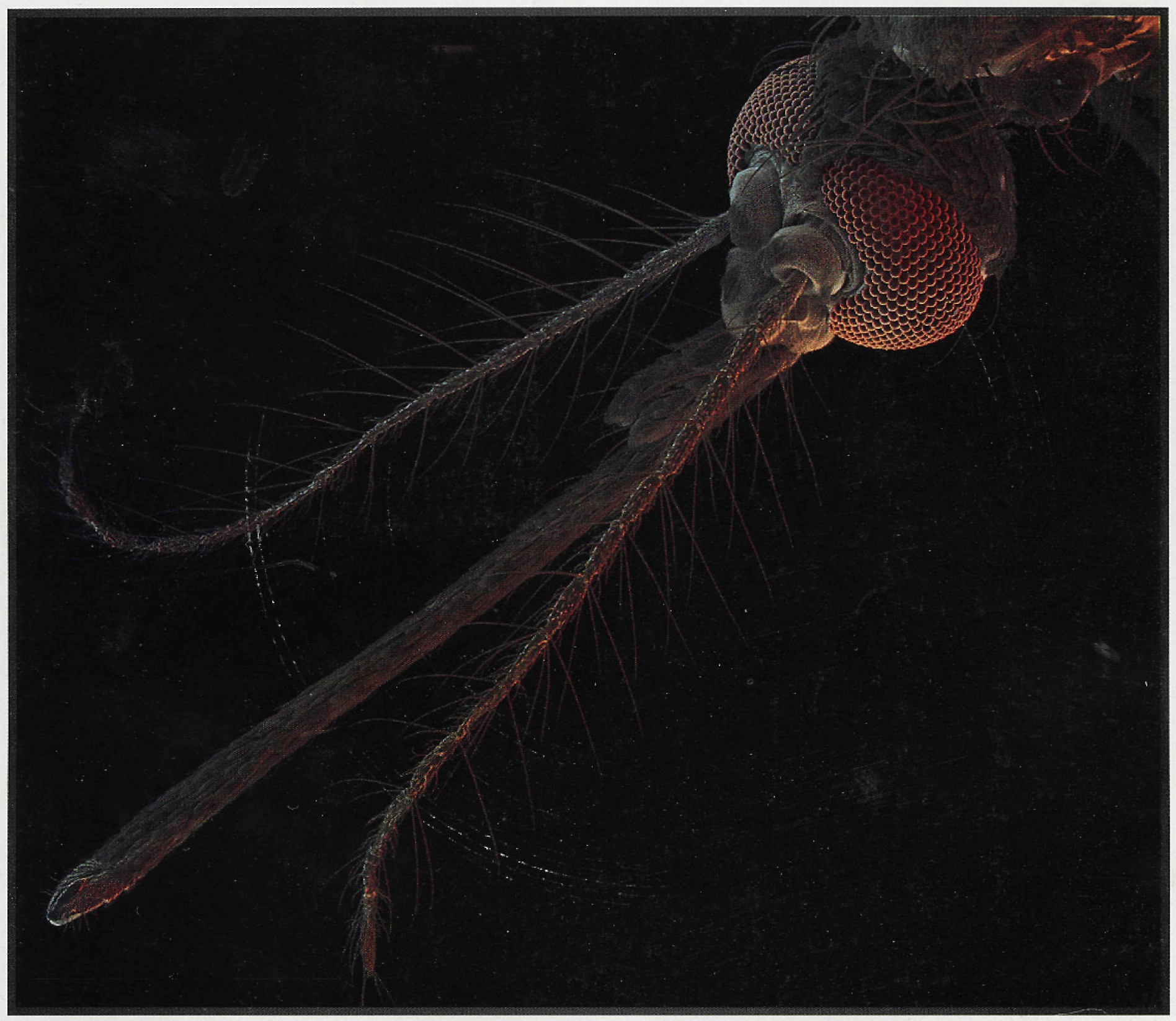




\section{Seeing is believing The LEO 1500 series is here...}

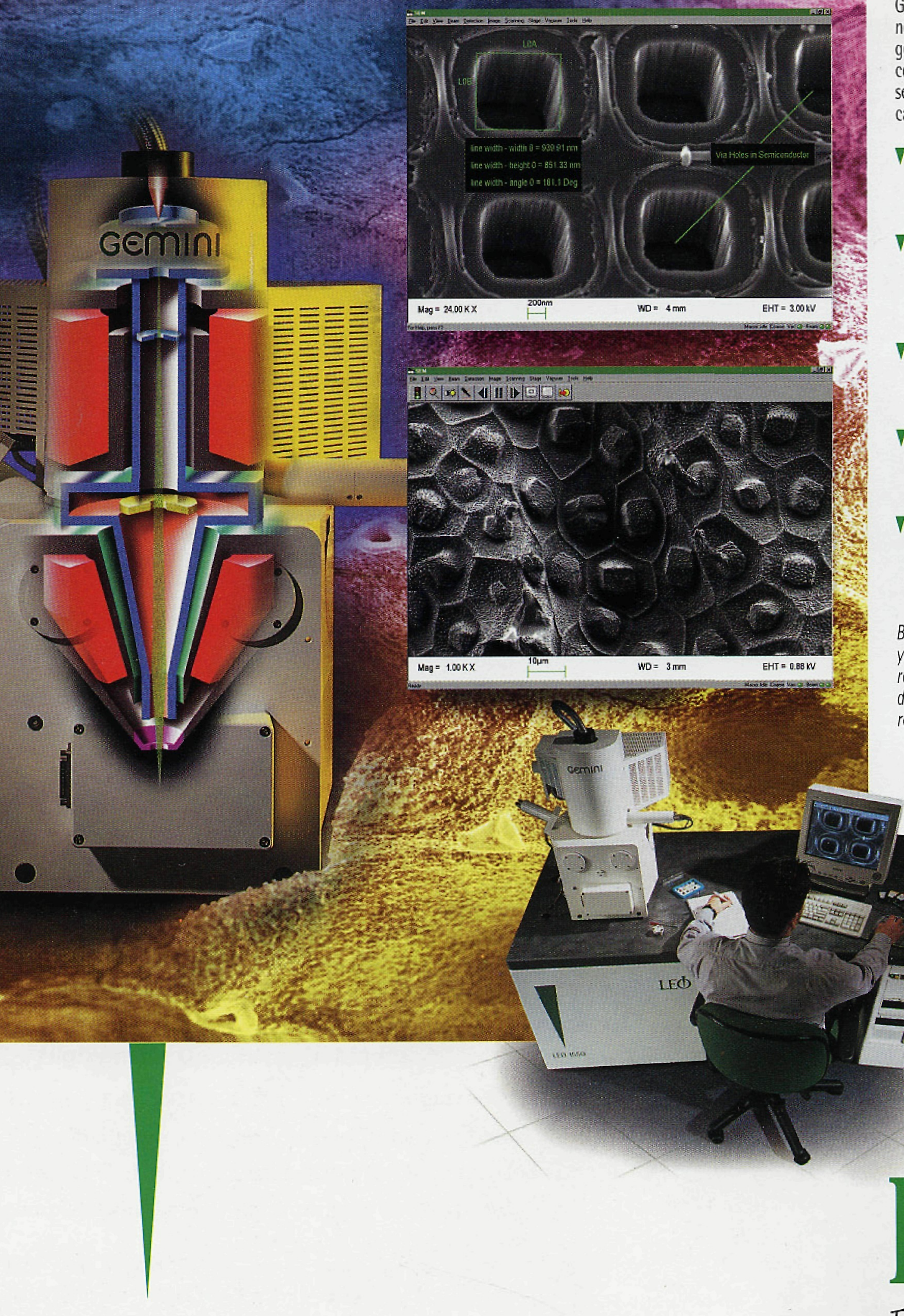

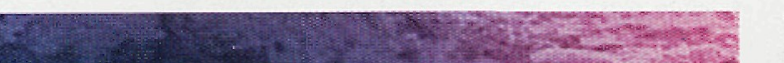

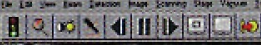

Set up different users' access levels, with a log-an procedure using the built-in Administrator facility.

7 Save images to disk or network at pixel resolutions up to $3 \mathrm{k} \times 2 \mathrm{k}$, 50 that you can even zoom the magnification on stored images.

$\nabla$ Choose the beam voltage (200V to $30 \mathrm{kV}$ ) to suit the sample or the analysis, because the resolution is superb at any voltage.

7 Fast and accurate analysis with EDX, EBSP, BSD thanks to the exceptionally stable high probe current.

$\checkmark$ Produce hard copy results in a format that you need using any Windows TI $^{\text {TI }}$ compatible printer - laser prints, colour dye subliminations, photographic etc.

Best of all, astonishing image quality is at your fingertips. Call your local LEO representative for information or a demonstration and you'll find that seeing really is believing.

LEO Electron Microscopy UK:

Telephone (44) 1223414166 Fax (44) 1223412776

E-mail info@leo-em.co.uk Website www.leo-em.ca.uk Germany:

Telephone (49) 7364946137 Fax (49) 7364944851 E-mail info@leo.de France:

Telephone (33) 141399210 Fax (33) 141399229 E-mail LEO_France@compuserve.com USA:

Telephone (1) 9147477700 Fax (1) 9146817443 Fax 70142504@compuserve.com Plus a worldwide network of dealers 


\section{TRAPPING SUBFEMTOLITER-SIZED VESICLES, AND ANALYZING THEM}

Stephen W. Carmichael, ${ }^{1}$ Mayo Clinic

The chemical analysis of secretory vesicles has long been limited by the fact that many vesicles are analyzed together, resulting in an averaging of a population. Differences between vesicles within that population could not be detected. Wouldn't it be great if vesicles could be analyzed one at a time? You guessed it, this breakthrough has been recently achieved.

The technique of capillary electrophoresis (CE) was modified by Daniel Chiu, Sheri Lillard, Richard Scheller, Richard Zare, Sandra Rodriguez-Cruz, Evan Williams, Owe Orwar, Mats Sandberg, and Anders Lundqvist so that vesicles could be probed singly. ${ }^{2}$ They pointed out that although we have been limited to analyzing populations of vesicles, there are examples in biology where the contents of a single vesicle is sufficient to elicit a response. But the problem remained of how to capture and analyze a vesicle in the femto- $\left(10^{-15}\right)$ to atto $\left(10^{-18}\right)$ liter size range.

It was demonstrated a few years ago that a single dye molecule could be detected by laser-induced fluorescence (LIF). However, detection is only the back half of the problem. The challenge that Chiu et al. addressed was the introduction and manipulation of an ultra-small sample. They chose the dense core vesicles from the atrial gland of the gastropod mollusk Aplysia califomica as the test sample. These easily-isolated vesicles are known to contain bioactive peptides that are involved in the animal's reproductive behavior. Careful analysis of a (relatively speaking) bulk sample with several available methods showed that these vesicles contained $\mathrm{NH}_{2}$-terminal peptides (NTPs), low-mass compounds (LMCs), and an unidentified peak (referred to as peak 4). Taurine was the main component of the LMCs, and they included leucine, isoleucine, and other modified or unusual amino acids.

To analyze a single vesicle, it was first optically trapped and a tapered capillary tip was moved into position next to it. The vesicle was about 0.5 microns in diameter, and the inside diameter of the tip was about ten times larger. A small voltage was applied which electrokinetically moved the vesicle into the tip. In video microscopic images published in their article, one could literally see the vesicle streaking into the capillary tip. With a single vesicle inside the tip, the capillary inlet was transferred into a small droplet of a reaction mixture containing naphthalene-2,3-dicarboxaldehyde (NDA) and potassium cyanide in borate buffer. A small amount of this reaction mixture is electrokinetically injected into the tapered inlet to lyse the vesicle. The lysate is reacted inside the capillary column with NDA, separated electrophoretically, and the components detected with LIF. Ten vesicles were individually analyzed in this fashion. They were all found to contain NTPs in roughly equal quantities, and either taurine or peak 4; taurine and peak 4 were never present in the same vesicle. When two or more vesicles were analyzed together, both taurine and peak 4 were detected. It was suggested that there could be two distinct population of vesicles (one containing taurine, the other peak 4), or a single population of vesicles whose composition changes with maturity.

Chiu et aL have developed a method that can separate and probe the chemical messages within single vesicles of only attoliters of volume. Whereas these vesicles from Aplysia were rather large, we can logically expect that the method will be refined so that smaller vesicles can be examined in the future, even to the zepto $\left(10^{-21}\right)$ liter range. This will make it possible to address many previously unanswerable biologic questions. The heterogeneity of vesicle populations is no longer an insurmountable problem to determining the composition of a single quantum of intercellular communication.

1 The author gratefully acknowledges Dr. Richard Zare for reviewing this article. 2 Chiu, D.T., S.J. Lillard, R.H. Scheller, R.N. Zare, S.E. Rodriguez-Crnz, E.R. Williams, W. Onvar, M. Sandberg. and J.A. Lundqvist. Probing single secretory vesicles with capillary electrophoresis. Science 279: 1190-1193. 1998.

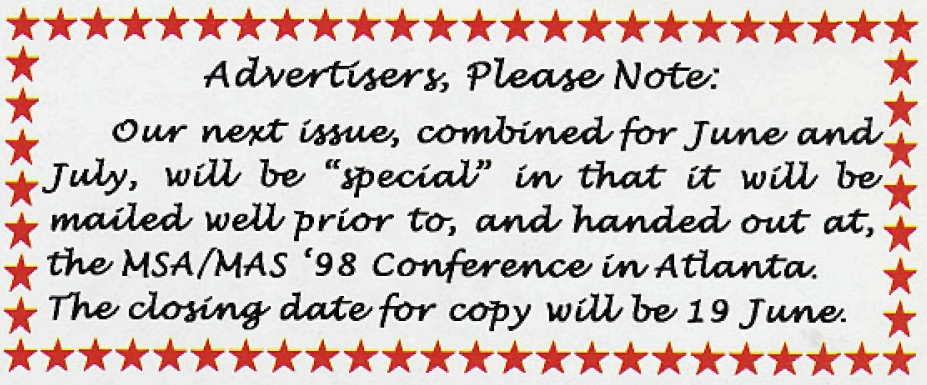

\section{Front Page Image SEM Photograph of a Mosquito - Female (Aedes Aegypti)}

Imaged on an ETEC SEM at $5 \mathrm{kV}$ using the SEM Wideband Multi-Detector Color Synthesizer (designed, built and patented by David Scharf). Then acquired digitally at 2,048 X 1,596 pixels directly into a Macintosh Power PC at a TIFF file, using Digital Micrograph software and Digiscan hardware. Then output to a CELCO film recorder, using Ektachrome 100+ film, to produce a $4 \times 5$ transparency.

Photograph is Copyright $\bigcirc$ by David Scharf, 1994

All Rights reserved.

This image is available at $250 \mathrm{X}$ as a $181 / 2^{\prime \prime} \times 19$ " high quality, lithographic print from Microscopy Today

For descriptions of all 16 images in the series, and NEW pricing, visit our web site:

http.//www.microscopy-today.com

\section{MIOROSCOPY TODAY}

A monthly newsletter dedicated to the unique interests in microscopy and mailed to some 8,000 microscopists worldwide at no cost in the U.S. and with a modest international charge.

PO Box 620122, Middleton, WI 53562 - Tel.: (608)836-1970 - Fax: (608)836-1969 - eMail: MicroToday@aol.com

http://www.microscopy-today.com

Copyright in 1997 by Today Enterprises. All rights teserved.

Don Grimes, Editor 